\title{
Diacronie
}

Studi di Storia Contemporanea

$\mathrm{N}^{\circ} 7,3 \mid 2011$

«Spagna Anno Zero»: la guerra come soluzione

\section{Rappresentazioni della Repubblica}

La Guerra e la Rivoluzione nelle memorie dei figli dei rifugiati spagnoli in Francia

Federica Luzi

\section{(2) OpenEdition}

Journals

\section{Edizione digitale}

URL: http://journals.openedition.org/diacronie/3376

DOI: 10.4000/diacronie.3376

ISSN: 2038-0925

Editore

Association culturelle Diacronie

Notizia bibliografica digitale

Federica Luzi, «Rappresentazioni della Repubblica », Diacronie [Online], № 7, 3 | 2011, documento 17, Messo online il 29 juillet 2011, consultato il 19 avril 2019. URL : http://journals.openedition.org/ diacronie/3376 ; DOI : 10.4000/diacronie.3376 


\section{Diacronie}

N. 7 | 7|2011 Spagna Anno Zero: la guerra come soluzione

$17 /$

Rappresentazioni della Repubblica,

la Guerra e la Rivoluzione nelle memorie dei

figli dei rifugiati spagnoli in Francia

Federica LUZI *

L'esilio spagnolo del 1939 in Francia fu un fenomeno che cambiò profondamente gli equilibri sociali del sud ovest del paese. Negli ultimi anni, le memorie trasmesse all'interno delle famiglie stanno diventando l'oggetto di numerose azioni associative $e$ di un forte investimento da parte delle istituzioni politiche locali. Nel 2009, in occasione del settantesimo anniversario dell'esilio, si è assistito ad un'eccezionale mobilitazione memoriale. I figli dei rifugiati impegnati nel lavoro di memoria, rivendicano questo passato come base della loro identità culturale e come eredità da valorizzare. Nella negoziazione dei significati dell'esperienza storica con le istituzioni politiche locali, le associazioni di discendenti elaborano delle rappresentazioni specifiche della Repubblica, la Guerra e la Rivoluzione, caricando alcuni avvenimenti di forti significati simbolici e rimuovendone altri. 


\section{Introduzione}

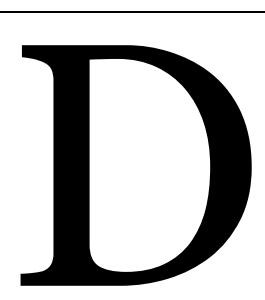

opo la caduta della Catalogna e la vittoria di Franco, nel gennaio 1939, circa cinquecento mila persone si diressero verso la frontiera francese, scappando dalle rappresaglie dell'esercito franchista. Accolti dalle autorità francesi nei campi di concentramento ${ }^{1}$ allestiti sulle spiagge del Roussillon, molti spagnoli sono rimasti in Francia partecipando alla Resistenza nel sud del paese ed integrandosi lentamente nella società francese. L'esilio di massa del 1939, per la lunga durata ${ }^{2}$ che lo caratterizzò, fu un evento eccezionale, che ebbe un impatto sociale molto profondo nel sud ovest della Francia.

La trasmissione della memoria storica e i racconti della Guerra, della Rivoluzione e dell'esilio sono rimasti per molti decenni custoditi all'interno delle famiglie dei rifugiati, delle reti di solidarietà spagnole e nei racconti, non sempre frequenti, dei testimoni.

Nel corso degli anni, alcune associazioni spagnole presenti sul territorio francese fin dal dopoguerra (come quelle degli anziani combattenti) hanno garantito, attraverso le commemorazioni di grandi personaggi politici spagnoli protagonisti della Guerra e della Resistenza, una qualche forma di trasmissione della memoria dei rifugiati. Si trattava, tuttavia, di pratiche commemorative legate al mondo militante spagnolo, che non faceva della memoria l'asse principale della propria attività. Alla fine degli anni Novanta, i discendenti dei rifugiati hanno iniziato a costituirsi in associazioni, con l'intento di lottare collettivamente contro l'oblio sociale al quale la memoria dei genitori sembrava condannata. Soltanto in coincidenza del sessantesimo anniversario dell'esilio, nel 1999, alcune nascenti associazioni di rifugiati di seconda generazione e qualche istituzione locale hanno iniziato a dare vita a una serie di iniziative pubbliche di commemorazione, che possiamo oggi identificare come la manifestazione embrionale di un movimento commemorativo che sarebbe esploso negli anni successivi.

1 Intorno alla questione terminologica si esercitano forti tensioni. I discendenti dei rifugiati spagnoli rivendicano la necessità di utilizzare il termine «concentramento» in riferito ai campi per spagnoli, mentre alcuni storici preferiscono utilizzare altre denominazioni come campi di «accoglienza» per non creare confusione con l'esperienza differente dei campi di concentramento nazisti. Scelgo di parlare di campi di «concentramento», poiché questa è la denominazione usata nei documenti amministrativi dal ministro dell'interno dell'epoca Albert Saurraut, per riferirsi ai campi per spagnoli distribuiti nel territorio francese.

$2 \quad$ Si può parlare di un fenomeno di lunga durata perché l'esilio finì soltanto circa quaranta anni dopo, con la caduta del franchismo. Per descrivere questo fenomeno complesso attraverso un'operazione di periodizzazione, riprendo la proposta della storica francese Geneviève Dreyfus-Armand, specialista dell'esilio spagnolo. La studiosa individua tre fasi politiche dell'esilio, che illustrano, in linea generale, le trasformazioni della presenza spagnola in Francia. La prima che va dal 1939 al 1944, la seconda dalla fine della guerra agli anni '6o e la terza dagli anni '6o ai giorni d'oggi. Cfr., DREYFUS-ARMAND, Geneviève, L'exil des républicains espagnols en France, de la guerre civil à la mort de Franco, Paris, Albin Michel, 1999. 
Questo fenomeno associativo si è sviluppato nelle tre regioni del sud ovest della Francia che sono state maggiormente segnate dalla storia dell'esilio (LanguedocRoussillon, Midy-Pyrénées e Aquitaine). Sotto l'impulso di questo movimento, anche le istituzioni politiche locali si sono progressivamente impegnate in questo lavoro di memoria.

Tuttavia, è nel 2009, settantesimo anniversario dell'esilio del 1939, che si è assistito a un'eccezionale mobilitazione associativa e istituzionale volta a riportare, nello spazio pubblico francese, la memoria dell'esilio spagnolo.

Questo risveglio memoriale inatteso coincide con la morte della gran parte di coloro che hanno vissuto questo dramma e la comparsa nello spazio pubblico dei loro figli, che rivendicano questo passato come una risorsa identitaria importante.

Attraverso le specifiche strategie culturali della memoria e dell'oblio, i discendenti dei rifugiati spagnoli sembrano ridefinire le basi delle proprie appartenenze. Rielaborare collettivamente la propria eredità storica significa recuperare o reinventare una sorta di sentire comune che leghi tutti gli individui, rendendoli capaci di pensarsi come un noi. Facendo leva sugli aspetti comuni dei percorsi individuali, le associazioni contribuiscono a costruire un nuovo spazio identitario dove riconoscersi ed essere riconosciuti, fornendo una rappresentazione di sé dotata di senso.

La rappresentazione della storia dei rifugiati che si sta imponendo nella società francese è dunque il prodotto di una negoziazione tra differenti promotori di memoria (le associazioni dei discendenti dei rifugiati e le istituzioni politiche locali). Attraverso le commemorazioni e l'insieme dei gesti che tramandano e conservano il passato, $i$ discendenti e le istituzioni costruiscono un discorso ufficiale. Esso è orientato a proporre e legittimare rappresentazioni specifiche di questo passato, che contengono giudizi sul presente e sul futuro e sono espressione degli interessi dei differenti soggetti.

È interessante, in particolare, riflettere su come alcuni degli eventi centrali della storia dei rifugiati spagnoli siano ricostruiti selettivamente e trasformati in rappresentazioni sociali. Si tratta, come afferma Teresa Grande riferendosi agli studi di Namer sulla commemorazione politica, di un «movimento soggettivo che va dal presente verso il passato»3. Le associazioni dei figli di rifugiati costruiscono i contenuti della memoria e forniscono nuove chiavi di lettura di alcuni grandi momenti del passato spagnolo.

In questo senso cercheremo di riflettere sul processo di affermazione delle nuove rappresentazioni sociali di tre momenti chiave delle vicende spagnole che sono al

3 GRANDE, Teresa, Le origini sociali della memoria, in TOTA, Anna Lisa (a cura di), La memoria contesa, Milano, Franco Angeli, 2001, p. 81. 
centro di questo processo di riappropriazione: la Repubblica, la Guerra e la Rivoluzione.

Come sono ricostruite le rappresentazioni dominanti di questi tre avvenimenti storici, dal valore simbolico e politico tanto differente? Attorno a quali universi di valori si cristallizzano queste rappresentazioni? Esistono delle sfumature d'interpretazione all'interno delle diverse arene di memoria: quella privata e familiare, quella collettiva (delle associazioni di discendenti) e quella istituzionale? Sulla base della definizione della sociologa Carmen Leccardi, si può parlare di memoria contesa, intesa come profondamente ambivalente poiché «mentre genera forti legami può fomentare divisioni altrettanto potenti e devastanti»4?

\section{La Guerra}

La Seconda Repubblica spagnola (1931-1939) è al centro del lavoro di memoria delle associazioni ed è uno dei temi principali dei discorsi commemorativi. È su di essa che i discendenti dei rifugiati spagnoli sembrano basarsi per reinventare e ricostruire la propria identità collettiva.

La Repubblica si è affermata negli anni come referente storico principale e svolge un ruolo centrale nel tentativo di creare un'immagine coerente e omogenea del gruppo di rifugiati spagnoli. Con il termine campo repubblicano i figli dei rifugiati raggruppano le differenti correnti ideologiche e politiche che animavano la politica spagnola del tempo, riuscendo così a smorzare i conflitti e creare un certo consenso. I figli dei rifugiati spagnoli hanno infatti conosciuto le profonde divergenze ideologiche che hanno animato i dibattiti e la vita politica dei genitori. Tuttavia, la seconda generazione ${ }^{5}$ mostra chiaramente la volontà di superare i conflitti interni e sottolinea la necessità di unirsi, al di là delle divergenze storiche, in nome di un dovere di memoria ${ }^{6}$. Come afferma Paloma Aguilar Fernández:

4 LECCARDI, Carmen, Prefazione, in TOTA, Anna Lisa (a cura di), La memoria contesa, Milano, Franco Angeli, 2001, p. 12.

$5 \quad$ Utilizzo la categoria di generazione, cosciente del fatto che spesso non restituisce la complessità del reale, poiché non tiene conto di altri elementi altrettanto importanti come la classe sociale, il livello culturale, etc.

$6 \quad$ Coniata nel secondo dopoguerra per esprimere la necessità etica di ricordare le atrocità del nazismo (Primo Levi la teorizza chiaramente nei numerosi libri dedicati al racconto della vita nei lager nazisti e della condizione esistenziale dei deportati), questa espressione è ormai abusata. Nei discorsi memoriali che invadono lo spazio pubblico europeo essa sembra essere divenuta un contenitore informe sul quale proiettare le necessità individuali e collettive di memoria, giustizia e riconoscenza. 
más allá de la incuestionable pluralidad de interpretaciones del pasado que pueda haber, lo importante es buscar, cuando exista, una interpretación del mismo suficientemente compartida, que tenderá por ello a ser muy general, subjetiva, ligada a intereses del presente ligeramente distinta en cada nueva evocación y con no pocos componentes mitológicos, algunos de los cuales, supuestamente, servirán para cohesionar al grupo 7.

La Repubblica sembra dunque rispondere a questa impellente necessità di coesione. Nelle venti interviste effettuate con figli di rifugiati spagnoli impegnati nel lavoro di conservazione e recupero della memoria, provenienti da famiglie comuniste, anarchiche, socialiste o repubblicane, tutti hanno sottolineato questo aspetto.

Emblematici sono a questi proposito i passaggi di due interviste; la prima realizzata con un figlio di anarchici e l'altra con un figlio di comunisti:

\begin{abstract}
...Alors de ce point de vue-là chez nous dans la génération des héritiers y’a quand même la volonté aussi de voir qu'est-ce qui était commun parce la guerre a été faite quand même ${ }^{8}$
\end{abstract}

...Nous on veut pas tomber là dedans, on est pas $1 \mid$ pour refaire la guerre d'Espagne, la guerre d'Espagne elle est faite, elle est finie, on est là maintenant pour que, que ça nous fasse plaisir ou pas, le travail de mémoire se fasse...9

Nel tentativo dei discendenti dei rifugiati di elaborare una rappresentazione collettiva e coerente della propria storia familiare, la Repubblica svolge un ruolo centrale. Essa rappresenta un tempo mitico, dove i valori universali di giustizia, libertà e uguaglianza regnano incorruttibili; la Repubblica sembra essere completamente idealizzata e collocata in una dimensione quasi onirica. Possiamo immaginare un parallelismo con il concetto di nostalgia strutturale elaborato dall'antropologo americano Michael Herzfeld, che la definisce come:

this collective representation of an Edenic order-a time before time-in which the balanced perfection of social relations has not yet suffered the decay that affects everything human. ${ }^{10}$

\footnotetext{
7 AGUILAR FERNÁNDEZ, Paloma, Políticas de la memoria y memorias de la política, Madrid, Alianza, 2008, p. 65.

$8 \quad$ Testimonianza di P.M. raccolta il 19 Maggio 2009 a Toulouse.

9 Testimonianza di A.M. raccolta il 30 Mai 2009 ad Agen.

10 HERZFELD, Michael, Cultural intimacy: social Poetics in the Nation-State, New York, Routledge, 1997, p. 109.
} 
Della Repubblica sono evidenziati gli aspetti più innovativi e progressisti e viene proposta come l'ideale politico di riferimento. Rappresenta un serbatoio di valori dal quale attingere per esprimere le proprie rivendicazioni presenti e su di essa è costruita una vera e propria iconografia della memoria. Il tricolore giallo-rosso-violetto, declinato in una miriade di gadget (orecchini, bandiere, coccarde, t-shirt etc..), in vendita durante le commemorazioni, sembra contribuire a naturalizzare ed essenzializzare la nuova identità collettiva dei figli dei rifugiati spagnoli.

La Repubblica, infatti, non è un relitto di un passato remoto, ma uno strumento per ripensare la propria esperienza e dar senso al presente. In questo senso sembra essere chiaramente investita da istanze e rivendicazioni politiche attuali. Questo elemento è ancora più chiaro alla luce delle commemorazioni dell'ottantesimo anniversario della Repubblica, celebrato quest'anno dalle associazioni dei discendenti ${ }^{11}$. Sembra delinearsi infatti, nella collaborazione sempre più stretta tra associazioni francesi e associazioni spagnole per la memoria storica, una certa volontà di agire sempre più attivamente, attraverso le battaglie di memoria, nella vita politica della Spagna, rivendicando a gran voce la necessità di abbandonare la monarchia e ritornare al modello repubblicano.

È necessario sottolineare, inoltre, come l’identificazione della storia dei rifugiati con quella della Repubblica faciliti la sua iscrizione nella società francese. Nella negoziazione costante di significati tra associazioni di discendenti e istituzioni politiche locali, che caratterizza questo processo di ricostruzione della memoria dei rifugiati spagnoli in Francia, la Repubblica rappresenta un elemento importantissimo. Come afferma Teresa Grande, «una memoria socialmente organizzata, per essere legittimata e accettata socialmente, deve necessariamente poggiare su qualcosa che è già in memoria sul sociale» ${ }^{12}$. L'assorbimento della memoria dei rifugiati spagnoli nella memoria nazionale francese è resa possibile attraverso l'esaltazione dei valori repubblicani, sufficientemente consensuali e generici. Nei discorsi dei presidenti delle associazioni e dei rappresentanti delle istituzioni locali tenuti durante le commemorazioni, la modernità della Seconda Repubblica spagnola è spesso messa in evidenza come elemento di continuità morale e ideologica con la storia francese ed europea.

11 La più grande e importante manifestazione di commemorazione della Repubblica spagnola in Francia del 2011 si è tenuta il 17 aprile scorso a Ille-sur-Têt, nel Pirenei Orientali. Hanno aderito e partecipato a questa iniziativa circa sessanta associazioni francesi e spagnole e più di mille persone provenienti dalla Francia, dalla Spagna, dal Messico e da altre parti del mondo.

12 GRANDE, Teresa, op. cit., p. 80. 
È però indispensabile distinguere tra una etero-rappresentazione, l'immagine cioè che i figli dei rifugiati trasmettono di sé all'esterno come gruppo, e l'auto-percezione, che include invece i contrasti e le tensioni interne.

Se l'immagine che le associazioni di discendenti vogliono proporre di sé all'esterno è quella di un gruppo coeso e unito, nelle storie di vita raccolte tra i discendenti è possibile individuare delle forme di resistenza all'omogeneizzazione delle memorie.

Tra i figli dei rifugiati anarchici, depositari di una memoria altra, la rappresentazione dominante della Repubblica come un passato idilliaco è spesso messa in discussione. Non tutti i discendenti, infatti, riconoscono il senso della propria identità familiare nell'ideale repubblicano. Dietro un'apparente accondiscendenza generale, se si escludono le commemorazioni organizzate dai diversi gruppi anarchici come la $\mathrm{CNT}^{13}$, che si pongono in netto contrasto alle associazioni nell'approccio alla memoria, si celano conflitti ideologici e racconti alternativi, che difficilmente trovano spazio. Nelle commemorazioni ufficiali, infatti, la pluralità delle memorie individuali e familiari sembra sacrificata in nome di una comune necessità di memoria, d'identità e di riconoscenza.

\section{Historiografía sobre les Brigadas Internacionales (1936-2010)}

I figli e le figlie dei rifugiati hanno un rapporto complesso con la memoria della Guerra, rapporto che porta con sé i fantasmi della morte e della violenza. All'interno delle famiglie sono molti i rifugiati che hanno scelto di non parlare della Guerra ai propri cari, consegnando così al silenzio le esperienze dolorose e traumatiche.

Nel lavoro di memoria dei discendenti attraverso le commemorazioni, il problema della ricostruzione di una rappresentazione globalmente accettabile dell'esperienza della Guerra e dell'immagine dei suoi protagonisti, sembra un tema molto importante e delicato.

Nonostante la difficoltà di attribuire un carattere completamente positivo al conflitto, che è spesso collegato nell'immaginario dei figli dei rifugiati al fratricidio e ai conflitti familiari ancora insoluti, esso trova la sua legittimità in una dimensione morale. La Guerra identifica dunque il mezzo estremo, legato a scelte storicamente determinate, per il raggiungimento di un ideale universale. Questo passaggio dal particolare al generale ha un significato profondo.

$13 \quad$ La CNT (Confederación Nacional del Trabajo) è la confederazione di sindacati anarchici spagnoli, che nacque a Barcellona nel 1910. Nell'esilio, la CNT spagnola si è riorganizzata in Francia. 
Le contingenze storiche e la specificità della situazione spagnola restano come sfondo ad una battaglia che assume sempre di più un carattere dicotomico e viene rappresentata come la Guerra del bene contro il male. L'impegno politico e militare degli spagnoli è pensato come una forma di chiaroveggenza politica. Nei discorsi commemorativi, infatti, viene stabilita una diretta continuità politica tra l’impegno militare contro l'ascesa del fascismo in Spagna e la partecipazione di molti spagnoli nel sud ovest della Francia alla Resistenza contro il nazismo. La rappresentazione della Guerra di Spagna come la prova generale della Seconda Guerra Mondiale è, come afferma lo storico François Godicheau:

(le) lieu commun le plus répété depuis la guerre elle-même, avant l’invasion de la Pologne par les armées de Hitler. Il s'agit en réalité d'une idée forgée pendant la guerre d'Espagne par la propagande républicaine à l'intention des opinions publiques des démocraties et en particulier de l'opinion publique britannique. ${ }^{14}$

Questa lettura degli eventi si sarebbe poi trasformata in uno dei topos narrativi più utilizzati per parlare della Guerra di Spagna. Godicheau, nell'articolo citato, sottolinea l'inesattezza di questa rappresentazione della Guerra, che è soltanto una delle possibili interpretazioni. Proposta da una parte degli attori del campo antifranchista nella seconda parte del conflitto, essa «ne saurait rendre compte de la complexité d'un affrontement protéiforme, qui dépasse la simple alternative fascisme/démocratie» ${ }^{15}$. Tuttavia, questa visione finalistica della storia contribuisce ad individuare, nelle scelte politiche e militari degli esiliati, un progetto di lotta coerente. I rifugiati spagnoli, sarebbero dunque stati i primi a comprendere la necessità di fermare ad ogni costo l'ascesa dei fascismi in Europa. In questo senso, la polemica sulla definizione del conflitto come una Guerra o una Rivoluzione sembra risolversi nell'idea di una guerra contro il fascismo. L'antifascismo fornisce una chiave di lettura privilegiata nella comprensione delle istanze politiche del fronte antifranchista. Questa rappresentazione della guerra permette inoltre di stabilire una continuità immaginata di valori tra la storia di cui sono depositari i figli dei rifugiati e la storia della Francia. Pensare i rifugiati spagnoli come degli eroi e come l'esempio del popolo francese durante la lotte per la liberazione, permette di inglobare la loro esperienza in quella della Resistenza, sulla quale lo stato francese ha costruito, dal dopoguerra, una forte retorica identitaria e nazionale. I discendenti possono dunque inscriversi pacificamente, con la propria

14 GODICHEAU, François, «"guerre civile”, "révolution", "répétition générale": les aspects de la guerre d'Espagne ", in BOURDERON, Roger (a cura di), La guerre d'Espagne. L'histoire, les lendemains, la mémoire, Paris, Tallandier, 2007, p. 90.

$15 \quad$ Ibidem, p. 91. 
eredità storica, accuratamente selezionata, nell'universo di senso rappresentato dalla storia nazionale.

Considerando che all'interno del tessuto associativo l'interpretazione della Guerra che abbiamo descritto è quella dominante e maggiormente condivisa, è interessante riflettere su come sia costruita, nei discorsi memoriali, l'immagine dei protagonisti del conflitto, combattenti e civili. Si può parlare della coesistenza di rappresentazioni differenti dei protagonisti della Guerra. I figli dei rifugiati alimentano il proprio immaginario del conflitto di racconti dalle tinte epiche che narrano i gesti eroici dei combattenti come esempi eccezionali di coraggio e di determinazione ${ }^{16}$. Questa immagine positiva risponde in parte alla necessità dei figli, come afferma Aline Angoustures, «de préserver l'image héroïque des pères» ${ }^{17}$. La visione eroica dei combattenti non è però esclusiva. Assistiamo infatti ad un doppio slittamento semantico: gli antifranchisti, condannati a restare nella storia come dei vinti, diventano, nei discorsi commemorativi dei presidenti delle associazioni e dei rappresentanti istituzionali, contemporaneamente dei vincitori e delle vittime.

L’immagine della Guerra sembra cristallizzarsi attorno a dei momenti particolari del conflitto, come la battaglia dell'Ebro. Nei racconti degli intervistati questa sconfitta terribile acquisisce un carattere drammaticamente mitico. Questa battaglia (25 luglio16 novembre 1938) costituì un momento significativo del conflitto ed ebbe delle conseguenze molto importanti. Quasi 200.000 soldati parteciparono agli scontri, 16.500 persone morirono e 64.000 restarono ferite. Come afferma l'antropologo Xavier Roigé, in un articolo sul processo di patrimonializzazione della battaglia dell'Ebro, riprendendo le riflessioni di Castell y Falcó:

En el Ebro, por tanto, se decidió el fin de la República española, pero al mismo tiempo representa un símbolo de la fuerte resistencia de los republicanos ante el avance franquista. La Batalla es, por tanto, un símbolo de la victoria para el franquismo, pero también una referenzia clara de de la cultura antifranquista democrática. ${ }^{18}$

È interessante riflettere sul fatto che gli intervistati, discendenti impegnati nel lavoro di
memoria, non specifichino se i genitori si siano arruolati dopo il 1937, forzatamente, o fossero
volontari. I figli sembrano voler pensare l'impegno militare come un atto volontario di coraggio;
in questo senso sottolineare la natura forzata dell'arruolamento potrebbe contribuire a
sminuirne il carattere eroico.
17 ANGOUSTURES, Aline, «Difficultés et paradoxes du devoir de mémoire, les enfants de
réfugiés espagnols en France», Matériaux pour l'histoire de notre temps «Espagne : la
mémoire retrouvée (1975-2002)», avril-juin 2003, n. 70, p. 15.
$18 \quad$ ROIGÉ, Xavier, «La patrimonializatión y musealización de los conflictos históricos.
Museos y espacios de la batalla del Ebro», in DEL MÁRMOL, Camila, FRIGOLÉ, Joan, 
Le associazioni francesi di discendenti sono solite organizzare pellegrinaggi collettivi nei luoghi della battaglia, investiti, negli ultimi anni, da un forte processo di patrimonializzazione, caratterizzato da una proliferazione locale di musei, di pubblicazioni di racconti e di memorie. Oltre la curiosità dei discendenti di ritornare sui luoghi del conflitto, consumando queste nuove forme di turismo storico e militante, la battaglia dell'Ebro resta nella memoria collettiva dei figli dei rifugiati come evento fortemente simbolico. Sembra infatti rispondere ad una doppia necessità rappresentativa. Da un lato, permette di ricordare gli antifranchisti spagnoli come combattenti valorosi, disposti all'estremo sacrificio per la realizzazione dei loro ideali, dall'altro di rappresentarli come vittime esemplari del fascismo, che di lì a poco avrebbe insanguinato l'Europa.

I combattenti vivono nella memoria collettiva, in questa dimensione epica dove l'immagine di vincitori e di vittime si alterna sullo sfondo degli ideali politici e del valore morale. La memoria della Guerra vissuta dalle donne, dai bambini e dai civili, assume invece una connotazione molto diversa. Essi sono rappresentati come vittime e la loro immagine non è mitizzata come quella maschile. Restano dunque nella memoria collettiva dei discendenti come i protagonisti silenziosi di alcuni eventi importanti della Guerra, come il bombardamento di Guernica ${ }^{19}$. Il coraggio delle donne e il loro ruolo centrale (nella Guerra e nell'esilio) viene piuttosto sottolineato nei racconti intimi e familiari; raramente è rivendicato pubblicamente. Per questo possiamo parlare di una memoria ufficiale della Guerra come una memoria di genere, in cui il ruolo delle donne è marginalizzato.

Utilizzando le parole di Johann Michel possiamo definire la rappresentazione della Repubblica come simbolo della lotta politica dei rifugiati spagnoli e del conflitto come Guerra per la difesa della democrazia delle «typifications réciproques d'échanges habituels de souvenirs» ${ }^{20}$. Sono cioè il prodotto di un lavoro di memoria attraverso cui ricordi genericamente condivisi e ritualizzati vengono assorbiti come dati oggettivi, diventando così verità storica.

NAROTZKY, Susana, (a cura di), Los lindes del patrimonio. Consumo y valores del pasado, Barcelona, Icaria, 2010, p. 218.

$19 \quad$ Il 26 aprile 1937, i franchisti, appoggiati dall'esercito hitleriano, misero in atto un feroce attacco alla popolazione, devastando la città basca. Questo evento ha acquisito una forte valenza simbolica anche grazie alla tela che Picasso, incaricato dal governo repubblicano, realizzò nel 1937.

${ }_{20}$ JOHANN, Michel, Gouverner les mémoires. Les politiques mémorielles en France, Paris, PUF, 2010, p. 13. 


\section{La rivoluzione}

Il dibattito sulla natura del conflitto e la diatriba Guerra o Rivoluzione è da sempre al centro dei discorsi dei protagonisti. Quasi tutti i discendenti intervistati raccontano gli interminabili scontri ideologici e terminologici tra spagnoli esiliati. La memoria della Rivoluzione, delle collettivizzazioni agrarie e della sperimentazione di nuove forme di organizzazione dell'economia locale è custodita nei racconti dei figli e delle figlie degli anarchici. Alcune associazioni memoriali costituite principalmente da figli di anarchici, o le organizzazioni anarchiche spagnole presenti sul territorio francese come la CNT, hanno organizzato negli anni numerose iniziative (in particolare a Toulouse e a Perpignan) per offrire un racconto storico delle esperienze rivoluzionarie vissute dai propri genitori.

Tuttavia, possiamo affermare che nella costruzione sociale di un racconto della storia dei rifugiati spagnoli, la Rivoluzione scompare. La volontà delle associazioni di discendenti di rappresentare, nei discorsi memoriali, il campo repubblicano come solidamente unito, innesca un forte processo di omologazione delle memorie. Questo non implica solamente un superamento delle differenze interne, ma una vera e propria selezione della storia da identificare come fondante della propria identità.

La memoria collettiva tende infatti a legittimare se stessa e ad assicurarsi il proprio predominio attraverso un utilizzo strumentale della memoria e della storia, considerando il passato in maniera selettiva. Come scrive Pedro Ruiz Torres:

seule la part du passé politiquement significative, au regard de la volonté de légitimer les identités et d'en assurer la domination, se transforme en héritage qui, bien entendu mérite d'être conservé et rappelé, au détriment d'une quantité d'autres expériences importantes qui malheureusement tombent dans l'oubli. ${ }^{21}$

Nell'iscrizione della storia dei rifugiati spagnoli nella società francese, gli aspetti più conflittuali e rivoluzionari della memoria degli esiliati sono taciuti.

L'oblio, come afferma Roger Bastide $^{22}$, è un vuoto pieno di qualche cosa; quest'assenza ha un significato profondo, poiché rappresenta una possibilità, per i figli dei rifugiati spagnoli, di costruire o restaurare un'immagine di sé globalmente soddisfacente. Le pratiche sociali della memoria, infatti, sono un lavoro di sintesi di una

$21 \quad$ RUIZ TORRES, Pedro, «Les usages politique de l'histoire en Espagne, formes, limites et contradictions», in HARTOG, François, REVEL, Jacques (a cura di), Les usages politique du passé, Paris, Éditions de l' École des Hautes Études en Sciences Sociales, 2001, p. 135.

${ }_{22}$ BASTIDE, Roger, «Mémoire collective et sociologie du bricolage», Bastidiana n. 7-8, 1994, pp. 209-242. 
pluralità di significati, che sono posti in ordine gerarchico. Nel momento in cui la seconda generazione decide di dedicarsi al lavoro di memoria, infatti, calibra le sue azioni anche nei confronti di un contesto sociale, quello francese, con il quale inizia una forma di negoziazione dei significati dell'esperienza storica stessa. La memoria della Guerra, della Rivoluzione e dell'esilio, potenzialmente scomoda per le autorità francesi, perché mette in luce gli errori politici della Francia ed è portatrice d'istanze rivoluzionarie, viene addomesticata e resa innocua.

Come scrive Paolo Jedlowski riferendosi ai processi di ricostruzione delle identità, esse «sembrano far leva sulla memoria in modo unilaterale: nel tentativo di cercare rinforzi simbolici per progetti politici in corso, lasciano da parte tutto ciò che di inquietante, problematico, sgradevole o oscuro può avere il passato» 23 . Parlare dell'oblio significa interrogarsi su come questo sia socialmente costruito, cercando di scoprire perché il gruppo sceglie una versione piuttosto che un'altra. La rivoluzione scompare nel silenzio e non trova spazio nelle narrazioni ufficiali della storia. Si parla di disordini piuttosto che di Rivoluzione, e l'esperienza delle collettivizzazioni agrarie è spesso messa sullo stesso piano delle uccisioni dei preti, dei padroni e dei carlisti. L'esperienza rivoluzionaria, quella anarchica in particolare, rappresenta un momento di rottura radicale nella storia europea ed è espressione di una serie di istanze sovversive come l'antistatalismo e l'autogestione, irriducibili ad un modello repubblicano o democratico.

I valori repubblicani ai quali le associazioni dei figli dei repubblicani spagnoli fanno riferimento nel raccontare la propria storia, sono, al contrario, valori condivisi, che possono essere rivendicati a gran voce da tutte le democrazie europee (e in modo particolare da quella francese), e trasversali, validi cioè al di là dei posizionamenti politici. La memoria collettiva dei figli dei rifugiati, allora, rinuncia agli aspetti più conflittuali, ma anche innovativi, della propria storia, allo scopo di trovare il proprio posizionamento nella storia ufficiale.

Ciò nonostante, l'osservazione partecipante durante le iniziative di commemorazione organizzate dai discendenti di rifugiati spagnoli, mi ha permesso di rilevare delle forme di resistenza a questo processo di omogeneizzazione delle memorie: ad esempio l'uso di diversi simboli e di diverse terminologie da parte dei figli degli anarchici durante una marcia simbolica o una conferenza, la loro assenza in alcune commemorazioni ufficiali o l'organizzazione di momenti in cui far vivere delle

23 JEDLOWSKI, Paolo, Memoria e interazioni sociali, in AGAZZI, Elena, FORTUNATI, Vita (a cura di), Memoria e saperi, percorsi transdisciplinari, Roma, Meltemi, 2007, p. 41. 
memorie alternative, come la commemorazione del 19 luglio a Toulouse ${ }^{24}$. Si tratta però di episodi marginali, che hanno poca risonanza pubblica. Il ruolo delle istituzioni politiche locali è infatti determinante nella costruzione delle rappresentazioni dominanti della storia dei rifugiati. Le associazioni di discendenti che collaborano costantemente con le istituzioni inscrivono il proprio lavoro nelle politiche memoriali ufficiali e godono di maggiore legittimità sociale rispetto ai gruppi che non rientrano in programmi istituzionali.

È così che nello spazio pubblico francese, in particolare nel sud ovest del paese, si stanno costruendo contemporaneamente dei vuoti memoriali rispetto all'esperienza rivoluzionaria e delle nuove rappresentazioni sociali della storia dei rifugiati spagnoli, che enfatizzano il loro eroismo nella Guerra, la loro sofferenza nell'esilio, il loro ruolo nella liberazione. Memoria e oblio sembrano essere entrambi funzionali alla reinvenzione di un'identità collettiva dei discendenti maggiormente implicati nel lavoro di memoria e alla costruzione di un discorso storico accettabile agli occhi delle istituzioni francesi ed europee.

Le rappresentazioni delle vicende storiche sulle quali abbiamo riflettuto nel presente articolo vanno pensate come il frutto di una negoziazione costante tra le necessità istituzionali di gestione delle politiche memoriali e le istanze identitarie collettive espresse dai discendenti, che cercano di ripensare la propria eredità storica e riscriverla in altri universi di significato.

Come scrive Godicheau, riflettendo sulla pluralità di denominazioni e di interpretazioni fornite dalle istituzioni e dalle organizzazioni durante il conflitto, «des hommes et des femmes adoptaient ces définitions pour donner sens à leur action» 25 . Tenendo conto della molteplicità dei punti di vista degli attori, però, sottolinea lo storico «aucune vision prétendument "objective" de cette guerre ne peut faire l'économie de ces sentiments collectifs» ${ }^{26}$.

Oggi, nel lavoro di memoria delle associazioni di discendenti, le rappresentazioni degli eventi sembrano farsi sempre più univoche e consensuali.

24 Il 19 luglio 1936, giorno in cui il governo repubblicano decide l'armamento del popolo e il 14 aprile 1931, giorno della proclamazione della Repubblica, sono due date particolarmente evocate nelle commemorazioni. A Toulouse, per molti anni, ogni anno gli esiliati spagnoli festeggiavano il 19 luglio con grandi incontri e feste. Oggi, per i discendenti dei rifugiati, scegliere di celebrare l'una o l'altra ricorrenza comporta un posizionamento ideologico molto diverso rispetto alla memoria. Le associazioni memoriali di discendenti tendono a dare maggior rilievo al 14 aprile, mentre i gruppi anarchici e i figli di rifugiati anarchici commemorano ancora oggi il 19 luglio.

25 GODICHEAU, François, op. cit., p. 98.

26 Ibidem, p. 98, (corsivo mio). 
In un contesto memoriale in cui collettivo e pubblico sembrano prendere il sopravvento sui sensi individuali, i racconti autobiografici raccolti nelle interviste sprigionano una forte carica eversiva. Come scrive l'etnologo Piero Vereni «volendo consegnare alla sedentarietà identitaria, il racconto (auto) biografico narra il proprio nomadismo, e nel farlo vanifica almeno l'ineluttabilità dell'identità» ${ }^{27}$.

Se alla società sono consegnate interpretazioni e rappresentazioni lineari e coerenti, altri universi di senso e altre interpretazioni sono possibili all'interno delle narrazioni individuali, che resistono e lottano contro l'omogeneizzazione delle esperienze.

\section{* L'autore}

Federica Luzi è dottoranda in Anthropologie Sociale et Ethnologie all’école des Hautes Études en Sciences Sociales a Parigi. Sotto la direzione di Daniel Fabre sta lavorando ad un progetto di tesi sulla riappropriazione e la rielaborazione della memoria dei rifugiati spagnoli in Francia. È membro del consiglio di amministrazione dell'associazione Adelante (associazione internazionale e pluridisciplinare di giovani ricercatori che lavorano sulla Guerra di Spagna e le sue ripercussioni).

URL: < http://studistorici.com/progett/autori/\#Luzi >

\section{Per citare questo articolo:}

LUZI, Federica, «Rappresentazioni della Repubblica, la Guerra e la Rivoluzione nelle memorie dei figli dei rifugiati spagnoli in Francia», Diacronie. Studi di Storia Contemporanea: Spagna Anno Zero: la guerra come soluzione, 29/07/2011,

URL:<:< http://www.studistorici.com/2011/07/29/luzi_numero_7>

Diacronie Studi di Storia Contemporanea $\mathcal{\beta}$ www.diacronie.it

Risorsa digitale indipendente a carattere storiografico. Uscita trimestrale. redazione.diacronie@hotmail.it

Comitato di redazione: Marco Abram - Giampaolo Amodei - Jacopo Bassi - Luca Bufarale - Alessandro Cattunar - Alice De Rensis Barbara Galimberti - Deborah Paci - Fausto Pietrancosta - Martina Sanna - Matteo Tomasoni - Luca Zuccolo 